\title{
Les archives de musique chinoise sortent des tiroirs
}

\section{François Picard}

\section{(2) OpenEdition}

\section{Journals}

Édition électronique

URL : http://journals.openedition.org/ethnomusicologie/639

ISSN : 2235-7688

\section{Éditeur}

ADEM - Ateliers d'ethnomusicologie

Édition imprimée

Date de publication : 1 novembre 2003

Pagination : 256-257

ISBN : 978-2-8257-0863-7

ISSN : $1662-372 X$

\section{Référence électronique}

François Picard, «Les archives de musique chinoise sortent des tiroirs », Cahiers d'ethnomusicologie

[En ligne], 16 | 2003, mis en ligne le 16 janvier 2012, consulté le 01 mai 2019. URL : http:// journals.openedition.org/ethnomusicologie/639

Ce document a été généré automatiquement le 1 mai 2019.

Tous droits réservés 


\title{
Les archives de musique chinoise sortent des tiroirs
}

\author{
François Picard
}

\section{RÉFÉRENCE}

Tudi yu ge. Songs of the Land in China. Labour Songs \& Love Songs, Qiao Jian-zhong, éd. 2 CD (70’37" + 68'58") Wind Records CB-08, Taipei, 1996. Livret en chinois 76 pages, en anglais 6 pages

Chuancheng. Yang Yinliu bainian danchen jinian zhuanji. Heritage. In Memory of a Chinese Music Master Yang Yin-Liu, Qiao Jian-zhong, éd. 2 CD (72'54" + 73'33") Wind Records CB-20 TCD 1023, Taipei, 2000. Livret en chinois 120 ages, en anglais 7 pages

1 Au moment où les ethnomusicologues français se penchent sur la question des archives, et en particulier sur la préservation de leurs propres enregistrements de terrain, il est sain de mettre en balance l'immense masse que constituent les archives d'une nation. La Chine a été touchée par l'enregistrement tout d'abord (années 1930) par l'intermédiaire des fabricants occidentaux de tourne-disques, devenus marchands de disques, puis (années 1940) par les premiers collecteurs, japonais. Le magnétophone à fil, puis à bande et enfin à cassettes, prend le relais, cette fois entre les mains des connaisseurs locaux.

Qiao Jianzhong, alors qu'il était directeur du prestigieux Institut de recherches musicales à Pékin, a eu la splendide initiative de publier - enfin - les enregistrements de terrain qui avaient servi de référence aux transcriptions sur lesquelles se sont basés, durant plusieurs décennies, tant les musicologues que les interprètes, sans que ces derniers puissent avoir accès au son lui-même. Bien sûr, les chanteurs d'opéra de Pékin comme Mei Lanfang ou de variétés comme Zhou Xuan avaient déjà fait l'objet de multiples rééditions dans tous les formats; mais le chant populaire des campagnes manquait cruellement. Cette lacune est enfin réparée, et offre l'occasion d'un véritable choc esthétique. Les enregistrements ici reportés datent principalement de la période des 
Cent-Fleurs (1956-1958) et de la brève accalmie (1963) qui a précédé l'orage de la Révolution culturelle.

3 Le volume consacré au plus grand musicologue que la Chine ait connu depuis quatre cents ans, Yang Yinliu (1899-1984), fondateur de l'Institut, montre deux qualités complémentaires de cet homme qui en possédait bien d'autres: «Ernest» Yang était en effet de confession protestante, et élevé dans la pratique de nombre d'instruments traditionnels de sa région, le Bas Yang-tse. Le premier $C D$ est consacré à ses transcriptions de partitions anciennes, essentiellement d'époques Song (Jiang Baishi, XIIe siècle) et Ming (Zhu Zaiyu, XVIIe siècle). Les arrangements et l'interprétation chorale ne correspondent certes pas à ce que ferait aujourd'hui une musicologie qui tirerait les leçons de la musique ancienne européenne, mais montrent une voie, hélas, désormais négligée vers une réappropriation des répertoires historiques transmis par l'écrit. Le second $\mathrm{CD}$ contient quant à lui des merveilles, des classiques inexpugnables, dont les mythiques et historiques enregistrements du seul génie dont la musique instrumentale chinoise ait gardé la mémoire enregistrée, Hua Yanjun, dit Abing, extraordinaire joueur de vièle erhu et de luth pipa, dont les quelques interprétations enregistrées et enfin publiées ici (du moins une pièce, la plus célèbre, plage 2) avaient été plagiées à satiété par cinq décennies d'interprètes professionnels. S'y ajoutent les tout aussi mythiques enregistrements de musiques de gongs et tambours, toujours de Wuxi, la ville natale de Yang Yinliu (plage 8). Regrettons cependant l'incomplétude de cette édition, qui se veut plus un hommage - mérité - au musicologue qu'une véritable édition des archives. Il existe sans doute encore d'autres enregistrements inédits d'Abing, et certainement d'autres de Wuxi (j'en ai en tout cas la copie chez moi...).

Ces enregistrements, déjà très largement documentés auparavant, sont servis par une édition de référence accompagnée de transcriptions et viennent rejoindre les opéras et les pièces instrumentales, en solo ou accompagnées, parues dans de nombreux repiquages, ainsi que les grandes anthologies et monographies des maîtres de cithare qin enfin largement publiées. Ils complètent de belle manière l'édition de musiques d'ensembles instrumentaux due à Stephen Jones en collaboration, déjà, avec Qiao Jianzhong.

\section{BIBLIOGRAPHIE}

Chine musique classique. CD Ocora C559039, 1988.

A Musical Anthology of the Orient, China, vol. 32. LP Musicaphon-Unesco BM.30.SL.2032 (1985); rééd. CD Unesco-Auvidis D 8071, 1996.

Zhongguo yinyue daquan, guqin juan. An Anthology of Chinese Traditional and Folk Music, A collection of music played on the Guqin. 8 CD China Record CCD-94/342 à 94/349, 1994.

Chine: Traditions populaires instrumentales. 2 CD AIMP, VDE-Gallo VDE-822/823, 1995.

Wu Jinglüe guqin yishu. The Qin Repertoire of Wu Jing-lue. 2 CDROI RB 001006-2C, 1998. 
Xu Yuanbai, Huang Xuehui Zhepai guqin yijun. The Qin Repertoire of Xu Yuan-bai \& Huang Xue-hui. 2 CD ROI RB 001012-2C, 2000.

Cai Deyun guyue yishu. Tsar Teh-yun The Art of Qin Music. 2 CD ROI RB 001006-2C, 2000. 